
\title{
Presercrch Suare \\ Hyperbolic graphene framework with optimum efficiency for conductive composites
}

\section{Xiaoting Liu}

Zhejiang University

\section{Kai Pang}

Zhejiang University

\section{Yingjun Liu}

Zhejiang University

\section{Chao Gao}

Zhejiang University https://orcid.org/0000-0002-3893-7224

Zhen Xu ( $\nabla$ zhenxu@zju.edu.cn )

Zhejiang University https://orcid.org/0000-0003-1352-9176

\section{Article}

Keywords: graphene framework, polymer composites, 2D sheets

Posted Date: July 13th, 2021

DOI: https://doi.org/10.21203/rs.3.rs-677007/v1

License: (c) (1) This work is licensed under a Creative Commons Attribution 4.0 International License. Read Full License 


\section{Abstract}

Constructing conductive filler networks with high efficiency is essential to fabricating functional polymer composites. Although two-dimensional (2D) sheets have prevailed in nanocomposites, their efficiency in enhancing conductive functions seems to reach the limit, as if merely addressing the dispersion homogeneity. Here, we exploit the unrecognized geometrical curvature of 2D sheets to break the efficiency limit of filler systems. The hyperbolic curvature meditates the incompatibility between 2D topology and 3D filler space and holds the efficient conductive path through face-to-face contact. The hyperbolic graphene framework exhibits the record efficiency in enhancing electrically and thermally conductive functions of nanocomposites. At volume loading of only $1.6 \%$, the thermal and electrical conductivities reach $31.6 \mathrm{~W} /(\mathrm{mK})$ and $13,911 \mathrm{~S} / \mathrm{m}$, respectively. Nanocomposites with hyperbolic graphene framework exhibit great potentials in thermal management, sensing and electromagnetic shielding. Our work presents a geometrically optimal filler system to break the efficiency limit of multifunctional nanocomposites and broadens the structural design space of 2D sheets by curvature modulation to meet more applications.

\section{Introduction}

The composites reflect the ancient wisdom of combining diverse properties into one component and play an increasingly important role in our modern technology ${ }^{1-3}$. In the very example of polymer nanocomposites, nanofillers with diverse topologies are introduced into polymeric matrices to enable rich functionalities of their nanocomposites ${ }^{4-6}$, for example, turning insulating into electrically and thermally conductive. The essential for preparing functional polymer composites is constructing efficient conductive fillers networks, that is, achieving the optimally high conductivities at the least containing of fillers. A natural deduction is that the geometric attribute of networks determines their efficiency. One traditional geometric concept of efficient networks is minimizing the percolation threshold ${ }^{4,7,8}$, at which nanofillers become continuous across the microscopic length (Fig. 1a, b). Most past efforts have followed the percolation concept, by choosing the topology of fillers from pseudo-zero dimensional particles to 1D nanowires and 2D sheets ${ }^{4,7-12}$. Beyond the sophisticated percolation notion, the efficiency of formed continuous networks should be considered as another important aspect to develop future functional nanocomposites. A scaling relationship $\sigma($ or $\mathbf{K}) \propto \beta \phi^{a}$ between functional properties and filler containing $(\phi)$ can be predicted for the fractal feature of networks, in which $\sigma$ or $\mathbf{K}$ are electrical or thermal conductivity and the $\beta$ and $a$ represent enhancement factor and filler efficiency. Following this relationship, higher $\beta$ and $a$ denote the higher enhancing efficiency of networks (Fig. 1b). Analyzing the ever-achieved advances ${ }^{4,6-12,13-21}$ demonstrates that $\beta_{\mathbf{K}}$ value has an increasing trend as the topology of nanofillers evolves from OD to 2D, but exhibits a limited range below 3 (Fig. 1C), which becomes a formidable limit for mixing filler system.

Graphene with 2D topology has emerged as a good nanofiller to improve the conductive functionalities of polymer composites for its outstanding electrical and thermal transport properties ${ }^{3}$. Following the 
traditional mixing protocol, graphene has exhibited high efficiency ${ }^{4}$ in enhancing conductive functions over other nanofillers, in the case of homogenous dispersion. To address the weak tunneling transport of polymer gaped connection, the continuous graphene network with direct connection ${ }^{22}$ and no insulating polymer separation has been utilized to promote the efficiency, following an alternative infiltration method to prepare composites. Although the direct contact path in graphene frameworks has extended the efficiency limit in the mixing protocol, the nearly line contact of graphene in polyhedral frameworks is not an ideally conductive path, which sets the new efficiency limit for 2D filler system with $\beta_{\mathbf{K}}$ below 10 (Fig. 1C) ${ }^{22-29}$. The limit of polyhedral card-house framework (Supplementary Fig. 1a) reflects the incompatibility between the Euclidean 2D topology and the occupying 3D non-Euclidean space, possibly originating from the narrow notion of flat consumption of 2D fillers. To further break this efficiency limit, it is reasonable to re-consider the geometric curvature of $2 \mathrm{D}$ sheets to construct more effective filler networks.

Here, we adopt a hyperbolic graphene continuous network as geometrically optimal filler to fabricate highly conductive polymer composites (PGC). The introduction of hyperbolic curvature of 2D sheets generates a close face-to-face connection configuration and increases the contact area between graphene layers, providing efficient conductive pathway for phonons and electrons. Hyperbolic graphene framework exhibits record efficiency in enhancing electrical and thermal conductivity, and breaks the previous efficiency limit of nanofillers ever achieved. The thermal and electrical conductivities of PGC reach $31.6 \mathrm{~W} /(\mathrm{mK})$ and $13,911 \mathrm{~S} / \mathrm{m}$ at a low containing of only $1.6 \mathrm{vol} \%$ of hyperbolic graphene network. The multifunctional PGC with hyperbolic graphene network exhibits highly mechanical flexibility, outstanding electromagnetic shielding property, and good thermal conductive capability.

\section{Results And Discussion}

\section{High enhancement efficiency of PGCs}

Hyperbolic graphene aerogel (HGA) was prepared by our hydroplastic foaming method ${ }^{30}$ from graphene oxide (GO) solid papers that consist of GO with average size of $\sim 50 \mu \mathrm{m}$ (Supplementary Fig. 2), followed by thermal reduction to restore the transport quality of graphene. The infiltration of polydimethylsiloxane (PDMS) into HGA got functional nanocomposites. We measured $\sigma$ and $\boldsymbol{K}$ of PGCs with a series of contents and assessed the efficiency parameter $(\beta)$ in the frame of scaling relationship. The $\beta$ values $\left(\beta_{\mathbf{K}}\right.$ and $\left.\beta_{\sigma}\right)$ in enhancing $\boldsymbol{K}$ and $\sigma$ of PGCs are 93 and $10^{15}$, respectively. The high efficiency in PGCs exceeds the efficiency ever-achieved in functional nanocomposites of diversely topologic nanofillers, as shown in Fig. 1c, Supplementary Fig. 3 and 4 . The $\beta_{\mathbf{K}}$ value of PGC (93) is nearly two orders of magnitude higher than that of the mixing composites, in which phonon transport is blocked by the gaped polymer between nanofillers. The polyhedral network of 2D sheets has exhibited $\beta_{\mathbf{K}}$ to approach $\sim 10$ in the GA composites, in which adjacent graphene sheets directly contact with each other to form continuous framework. By comparison, the PGC has a breakthrough efficiency, achieving nearly one order of magnitude higher than that of nanocomposites with polyhedral network. In the geometric principle, the 
hyperbolic network forms face-to-face contact of 2D sheets and provides much more conductive paths than that of polyhedral network with the line-line contact configuration. The PGC introduces a new concept of curvature to reconcile the geometric incompatibility of 2D sheets with the occupied 3D space.

\section{Morphology and properties of HGAs}

Structural analysis reveals the hyperbolic geometry of hydroplatically foamed GAs, featuring face-to-face contact of curved graphene sheets (Fig. 2a-e and Supplementary Fig. 5). The hyperbolically curved conformation of graphene sheets is generated by the bubbling mechanism of hydroplastic foaming, in which GO laminates adhere to the bubble surface and form facial tight connection by capillary force. Xray computed microtomography analysis reveals the hyperbolically curved structure of hydroplastic foaming GA, appearing a homogenous porous structure in the long millimeter scale (Fig. 2a). Scanning electron microscope (SEM) inspection demonstrates hyperbolically curved conformation of graphene sheets, including sphere surface and undulating edge. The curved graphene sheets warp together to form porous structure with a spherical diameter $(D)$ ranging from 15 to $200 \mu \mathrm{m}$, corresponding to a density $(\rho)$ of 5 to $40 \mathrm{mg} / \mathrm{cm}^{3}$, following the relationship of $\rho=263.14 D^{0.710}$ (Supplementary Fig. 6), close to the theoretical relation of $\rho \sim D^{-1}$ (ref. 30). The structural analysis allows the depiction of the 3D structural model of HGA (Fig. 2d). The hyperbolic curvature of graphene sheets permits the characteristic facial contact mode (Fig. 2c, e), and the contact area is estimated to occupy nearly $10-25 \%$ of the surface area of curved graphene sheets (Supplementary Fig. 7). This face-to-face contact mode is different from the line-line contact in the polyhedral GAs and enables much broader conductive paths from the theoretical analysis (Fig. 2h, Supplementary Fig. 1, 8 and 9). After thermal annealing up to 2,800 ${ }^{\circ} \mathrm{C}, \mathrm{GO}$ completely transforms to graphene through decomposition of functional groups and recrystallization. The annealed graphene sheets restore the high crystalline quality of graphene lattice and have neglectable structural defects, as confirmed by the long-range crystalline order in high resolution transmission electron microscopy (HRTEM) inspection (Fig. 2f, g) and the trace D peak in Raman spectra (Supplementary Fig. $10)$, as well as X-ray diffraction (XRD) and X-ray photoelectron spectroscopy (XPS) results (Supplementary Fig. 11).

The continuous hyperbolic network of highly crystalline graphene sheets enables the extraordinary electrical and thermal conductivity of GAs, outperforming the pervious neat GAs fabricated by freezedrying and even templated CVD growth (Fig. $2 \mathrm{i}$ and j, Supplementary Fig. 12 and 13). The $\sigma$ value of HGA along the facial direction gradually increases from $1,304 \mathrm{~S} / \mathrm{m}$ at $\rho$ of $4.3 \mathrm{mg} / \mathrm{cm}^{3}$ to $10,703 \mathrm{~S} / \mathrm{m}$ at $\rho$ of $39.1 \mathrm{mg} / \mathrm{cm}^{3}$, following a scaling relation of $\sigma \sim \rho^{1.0}$ (Fig. 2i and Supplementary Fig. 12a). At a given density, HGAs have a higher $\sigma$ as compared with previously reported $\mathrm{GAs}^{22,31-36}$. For example, the $\sigma$ of $8,576 \mathrm{~S} / \mathrm{m}$ of $\mathrm{HGA}$ is $166 \%$ higher than that of the CVD-grown GA $(3,229 \mathrm{~S} / \mathrm{m})^{22}$ at an approximately same $\rho$ of $30 \mathrm{mg} / \mathrm{cm}^{3}$. More striking is the high thermal conductivity of $\mathrm{HGA}^{37-41}$. The thermal diffusion coefficient in the facial direction gradually increases to $\sim 662 \mathrm{~mm}^{2} / \mathrm{s}$ at $39.9 \mathrm{mg} / \mathrm{cm}^{3}$, following a scaling law of $\boldsymbol{K} \sim \rho^{1.6}$ (Fig. 2j and Supplementary Fig. 12b). The highest $\boldsymbol{K}(18.76 \mathrm{~W} /(\mathrm{mK}))$ of HGA achieved is $494 \%$ higher than the freezing dried GA $(3.16 \mathrm{~W} /(\mathrm{mK}))$ after the same heat annealing (Supplementary Fig. 
14). By a systematic comparison, the electrically and thermally conductive properties of HGA reach the record efficiency to outperform other carbon networks. This superior efficiency is rooted from the hyperbolically curved conformations of graphene sheets with tight facial contact and broader conductive paths.

\section{HGA filled polymer nanocomposites}

The neat HGA provides an optimally efficient filler network to improve the conductive properties of composites. We prepared the model PGCs by vacuum infiltrating precursors followed by thermal curing (Fig. 3a). The large-area PGCs can be facilely prepared in batches, as shown as the composite mat with an area of $\sim 1,500 \mathrm{~cm}^{2}$ (Fig. 3b and Supplementary Fig. 15). The hyperbolically curved structure was maintained during the filtration and curing processes. SEM inspections reveal that the graphene network in the composite maintains the spherical shape on the surface and keeps indulging continuous connections on the section (Fig. 3c and Supplementary Fig. 16), that is similar to the structure of neat HGA (Fig. 2a-c).

The model PGC exhibits an overwhelmingly high filler efficiency in enhancing thermal and electrical conductivity, outperforming the efficiency of composites with nanofillers extending from OD particles, 1D nanowires, 2D sheets to conventional GAs (Fig. 3d and Supplementary Fig. 17). For all nanofillers, the $\boldsymbol{K}$ monotonously increases as the filler content increases. But the PGCs follow the steepest trend and have the highest thermal conductivity at the same filler content, exhibiting the highest efficiency than any other filler composites $4,6-9,11,16,22,23,25$. For example, the $\boldsymbol{K}$ of PGC achieves $31.6 \mathrm{~W} /(\mathrm{mK})$ only at a containing of $1.6 \%$, and the peak value is $5,40,60$ and 150 folds higher than those of $3 \mathrm{D}$ GAs $(\sim 6 \mathrm{~W} /(\mathrm{mK})), 2 \mathrm{D}$ graphene $(0.8 \mathrm{~W} /(\mathrm{mK}))$, $1 \mathrm{D}$ carbon nanotube $(\mathrm{CNT}, 0.5 \mathrm{~W} /(\mathrm{mK}))$ and $0 \mathrm{D}$ carbon black $(0.2 \mathrm{~W} /(\mathrm{mK}))$, respectively. More impressively, the PGC with optimized geometrical structure shows an ultrahigh thermal conductivity enhancement (TCE) of $15,700 \%$, which is calculated by $\times 100 \%$, where and are the thermal conductivity of composite and matrix, respectively (Supplementary Fig. 18), corresponding to the specific TCE (TCE per 1 vol\% filler addition) of $9,813 \%$. Beside the predominant $\boldsymbol{K}$, the PGCs also exhibit overwhelmingly high $\sigma$ values, and the data line is located at the top to break through the limit of previous composites ${ }^{5,22,42-53}$. The highest $\sigma$ of PGCs reaches $13,911 \mathrm{~S} / \mathrm{m}$ at the containing of $1.6 \mathrm{vol} \%$, outperforming GAs with polyhedral network and other nanofiller systems.

\section{Electromagnetic properties of flexible PGCs}

The PGC bulks remain high resilience as the pristine PDMS elastomer, under stretching, bending and compression. The tensile strength of PGC barely decays and loading-unloading curves are almost overlapped for 1,000 cycles at a strain of 10\% (Fig. 4a). The cycle bending test at small bending radius reveals the stable bending elasticity (Fig. 4b). As to the compression, the PGC recovers to its original height at high strain of $20 \%$ for 1,000 cycles (Fig. $4 \mathrm{c}$ and Supplementary Fig. 19). Besides, PGC behaves great sensitivity to these mechanical behaviors (Supplementary Fig. 20). 
The high electrical conductivity of PGC enables itself as a predominant electromagnetic interference (EMI) shielding material. With the increase of the graphene content from $0.17 \%$ to $0.98 \%$, the average shielding effectiveness $(S E)$ of PGCs increases from $\sim 47 \mathrm{~dB}$ to $\sim 100 \mathrm{~dB}$ in the frequency range of 2-18 $\mathrm{GHz}$, far exceeding the commercial standard of $20 \mathrm{~dB}$ (Fig. $4 \mathrm{~d}$ and Supplementary Fig. 21a). In addition, the EMI shielding performance of PGC almost keeps stable after 1,000 bending cycles (Supplementary Fig. 21 b), only with a reduction by $\sim 5 \mathrm{~dB}$. The superior efficiency in electrically conductive PGC allows the superiority in EMI shielding. As shown in Fig. 4e, the PGC performs better than previously reported EMI shielding composites containing other nanofillers, such as MXenes ${ }^{54-57}$, CNTs ${ }^{58-61}$, Ag nanowires $(A g N W s)^{62-64}$ and ordinary GAs ${ }^{49,53,65-68}$, providing an ideally functional materials for electromagnetic environmental protection.

\section{Thermal management of flexible PGC}

The overwhelming thermal conductivity of PGC with the least filling content provides new opportunities for thermal management, especially for high power electronics. As a proof, we use the PGC mat with 0.2 vol\% graphene loading as heat diffusion and heat transfer materials for a high-power LED lamp (Fig. 5a). For heat diffusion, a high-power LED was placed on the pure PDMS (as a contrast) and PGC (Supplementary Movie 1). As displayed in Fig. 5b and c, the LED lamp equipped with the PGC mat as a thermal spreader exhibit lower heat-spot temperature than pure PDMS after $180 \mathrm{~s}$ work. For heat transfer, PGC and PDMS were vertically put between a high-power LED and an aluminum heat sink, respectively. The flexible thermal interfacial material of PGC can effectively transfer the heat to the bottom heat sink (Supplementary Movie 2), which brings dramatically temperature drop in the LED lamp (Fig. 5d, e). These results indicate the as-prepared flexible PGC not only holds higher thermal conductivity, but also exhibits great potential to relieve the urgently thermal management demands in electronic devices.

\section{Conclusions}

We have developed a hyperbolic graphene continuous network as a geometrically optimal filler to achieve the record efficiency in enhancing conductive properties of nanocomposites. The introduction of curvature design breaks the efficiency limit of 2D lamellar filler and extends the neglected parameter space of topologic curvature. The experimental and theoretical analysis reveal that the high efficiency roots from the intrinsic face-to-face connections of hyperbolically curved graphene networks, which provide more conductive pathways for phonons and electrons. The enhancement factors of thermal $\left(\beta_{\mathbf{K}}=93\right)$ and electrical $\left(\beta_{\sigma}=10^{15}\right)$ conductivity outperform previous $0 \mathrm{D}, 1 \mathrm{D}, 2 \mathrm{D}$ and 3D functional nanofillers for polymer composites. The polymer composites with hyperbolic graphene networks exhibit high thermal $(31.6 \mathrm{~W} /(\mathrm{mK}))$ and electrical $(13,911 \mathrm{~S} / \mathrm{m})$ conductivities at an exceptionally low volume fraction of only $1.6 \%$. We have revealed the great values of polymer composites containing hyperbolic fillers in electromagnetic shielding and thermal management. The hyperbolically curved graphene network opens the curvature design of 2D sheets and other fillers, guides the structural design of functional composites and promotes more functional applications in sensors and energy storage. 


\section{Materials And Methods}

Preparation of HGAs. Aqueous GO dispersions with the average size of $~ 50 \mu \mathrm{m}$ were produced from Hangzhou Gaoxi Technology Co. Ltd. (www.gaoxitech.com). Self-supporting GO film was acquired by casting GO dispersions onto the PET substrate. Then the dried GO films were soaked into $10 \% \mathrm{~N}_{2} \mathrm{H}_{4}$ aqueous solution to realize hydroplastic foaming $\mathrm{GAs}^{30}$. The GA density can be tuned by the foaming time. The HI/HAc (1:1) reduction and high-temperature treatment $\left(2,800^{\circ} \mathrm{C}\right.$ for $\left.2 \mathrm{~h}\right)$ were conducted to obtain conductive HGAs.

Preparation of PGCs. A typical vacuum impregnant method in industrial engineering was adopted for the fabrication of polymer composites. As-prepared HGAs with different densities were placed into the PDMS prepolymer, followed by degassing in a vacuum environment for $30 \mathrm{~min}$ and thermally curing at $60^{\circ} \mathrm{C}$ for 10 min. Then PGCs with various graphene loadings were achieved.

Characterizations. The morphology and microstructure of HGAs and PGCs were characterized by fieldemission SEM (Hitachi S4800), TEM (FEI Titan G2 60-300) and CT with a resolution of $200 \mathrm{~nm}$ (skysCan2211). Raman measurements were performed on a Renishaw inVia-Reflex using a $532 \mathrm{~nm}$ laser beam. XPS characterization was conducted using an Escalab 250Xi instrument. XRD patterns was measured with a X-pert Powder diffractometer. The compressive test was performed on a microcomputer control electronic universal testing machine (RGWT-4000-20, REGER). The tensile and bending measurements were used by the Instron Legend 2344 machine. The electrical properties of HGAs and PGCs were evaluated by the Keithley 2400 Source Meter. The EMI shielding performances were measured by Vector Network Analyzer (ZNB40). The thermal diffusivities were directly measured using the LFA 467 HyperFlash. The thermal conductivity was calculated using the equation $\mathbf{K}=a \times C_{\mathrm{p}} \times \rho$, where $a$ is the thermal diffusivity, $C_{\mathrm{p}}$ is the specific heat capacity, and $\rho$ is the density of the sample. The heat capacity $\left(C_{\mathrm{p}}\right)$ was measured by DSC (TA Q200) at a heating rate of $10^{\circ} \mathrm{C} / \mathrm{min}$ under nitrogen atmosphere. The IR thermal photos were captured by a high-resolution infrared imager ((FLIR T630sc).

\section{Declarations}

\section{Acknowledgments}

This work is supported by the National Natural Science Foundation of China (Nos. 51973191, 52090030 and 51803177), National Key R\&D Program of China (No. 2016YFA0200200), Hundred Talents Program of Zhejiang University (188020*194231701/113), Key research and development plan of Zhejiang Province (2018C01049), Fujian Provincial Science and Technology Major Projects (NO. 2018HZ0001-2), the Fundamental Research Funds for the Central Universities (Nos. K20200060 and 2021FZZX001-17), Key Laboratory of Novel Adsorption and Separation Materials and Application Technology of Zhejiang Province (512301-I21502) and Devices of the Ministry of Education NJ2020003 (INMD-2021M06).

\section{Author contributions}


Z. X., Y. J. L. and C. G. conceived the research. Z. X., X. T. L. and K. P. designed experiments, analyzed the data and wrote the manuscript. X. T. L. conducted the literature investigation and thermal and electrical performance tests. X. T. L. and K. P. did the mechanical and electromagnetic shielding tests of PGCs and application presentations.

\section{Competing interests}

The authors declare no competing interests.

\section{Data availability}

The data that support the findings of this study are available upon reasonable request from the corresponding authors.

\section{Additional information}

Supplementary Information accompanies this paper.

Correspondence and requests for materials should be addressed to Z. X.

\section{References}

1. Yuan, K. J. et al. Engineering the thermal conductivity of functional phase-change materials for heat energy conversion, storage, and utilization. Adv. Funct. Mater. 30, 1904228 (2020).

2. Huang, X. Y. et al. Thermal conductivity of graphene-based polymer nanocomposites. Mater. Sci. Eng. $R$ 142,100577 (2020).

3. Sun, X. X et al. Recent progress in graphene/polymer nanocomposites. Adv. Mater. 33, 2001105 (2020).

4. Yu, A. P., Ramesh, P., Itkis, M. E., Bekyarova, E. \& Haddon, R. C. Graphite nanoplatelet-epoxy composite thermal interface materials. J. Phys. Chem. C111, 7565-7569 (2007).

5. Naficy, S. \& Garmabi, H. Study of the effective parameters on mechanical and electrical properties of carbon black filled PP/PA6 microfibrillar composites. Compos. Sci. Technol. 67, 3233-3241 (2007).

6. Sun, P. C. et al. Room temperature electrical and thermal switching CNT/hexadecane composites. Adv. Mater. 25, 4938-4943 (2013).

7. Ram, R., Soni, V. \& Khastgir, D. Electrical and thermal conductivity of polyvinylidene fluoride (PVDF)conducting carbon black (CCB) composites: Validation of various theoretical models. Composites Part $B$. 185,107748 (2020).

8. Bonnet, P., Sireude, D., Garnier, B. \& Chauvet, O. Thermal properties and percolation in carbon nanotubepolymer composites. Appl. Phys. Lett. 91, 201910 (2007). 
9. Hong, J., Park, D. W. \& Shim, S. E. Electrical, thermal, and rheological properties of carbon black and carbon nanotube dual filler-incorporated poly(dimethylsiloxane) nanocomposites. Macromol. Res. 20, 465-472 (2012).

10. Song, J. P., Tian, K. Y., Ma, L. X., Li, W. \& Yao, S. C. The effect of carbon black morphology to the thermal conductivity of natural rubber composites. Int. J. Heat Mass Transfer 137, 184-191 (2019).

11. Song, S. H. et al. Enhanced thermal conductivity of epoxy-graphene composites by using nonoxidized graphene flakes with non-covalent functionalization. Adv. Mater. 25, 732-737 (2013).

12. Xue, Q. Z. Model for the effective thermal conductivity of carbon nanotube composites. Nanotechnology 17, 1655-1660 (2006).

13. Moisala, A., Li, Q., Kinloch, I. A. \& Windle, A. H. Thermal and electrical conductivity of single- and multiwalled carbon nanotube-epoxy composites. Compos. Sci. Technol. 66, 1285-1288 (2006).

14. Gojny, F. H. et al. Evaluation and identification of electrical and thermal conduction mechanisms in carbon nanotube/epoxy composites. Polymer 47, 2036-2045 (2006).

15 Shahil, K. M. F. \& Balandin, A. A. Graphene-multilayer graphene nanocomposites as highly efficient thermal interface materials. Nano Lett. 12, 861-867 (2012).

16. Alam, F. E. et al. In-situ formation of cellular graphene framework in thermoplastic composites leading to superior thermal conductivity. J. Mater. Chem. A 5, 6164-6169 (2017).

17. Gu, J. W. et al. Thermal percolation behavior of graphene nanoplatelets/polyphenylene sulfide thermal conductivity Composites. Polym. Compos. 35, 1087-1092 (2014).

18. Xin, G. Q. et al. Advanced phase change composite by thermally annealed defect-free graphene for thermal energy storage. ACS Appl. Mater. Interfaces 6, 15262-15271 (2014).

19. Yang, B. et al. Filler network structure in graphene nanoplatelet (GNP)-filled polymethyl methacrylate (PMMA) composites: From thermorheology to electrically and thermally conductive properties. Polym. Test. 89, 106575 (2020).

20. Song, N. et al. Highly thermally conductive polypropylene/graphene composites for thermal Management. Composites Part A 135, 105912 (2020).

21. Huang, J. R. et al. Massive enhancement in the thermal conductivity of polymer composites by trapping graphene at the interface of a polymer blend. Compos. Sci. Technol. 129, 160-165 (2016).

22. Shen, X. et al. Three-dimensional multilayer graphene web for polymer nanocomposites with exceptional transport properties and fracture resistance. Mater. Horiz. 5, 275-284 (2018). 
23. Lian, G. et al. Vertically aligned and interconnected graphene networks for high thermal conductivity of epoxy composites with ultralow loading. Chem. Mater. 28, 6096-6104 (2016).

24. Yang, J. et al. Air-dried, high-density graphene hybrid aerogels for phase change composites with exceptional thermal conductivity and shape stability. J. Mater. Chem. A 4, 18067-18074 (2016).

25. Yang, J. et al. High-quality graphene aerogels for thermally conductive phase change composites with excellent shape stability. J. Mater. Chem. A 6, 5880-5886 (2018).

26. Yang, J. et al. Hybrid graphene aerogels/phase change material composites: Thermal conductivity, shape-stabilization and light-to-thermal energy storage. Carbon 100, 693-702 (2016).

27. Liu, Z. D. et al. Graphene foam-embedded epoxy composites with significant thermal conductivity enhancement. Nanoscale, 11, 17600-17606 (2019).

28. Li, Y. et al. Construction of highly aligned graphene-based aerogels and their epoxy composites towards high thermal conductivity. J. Mater. Chem. C7, 11783-11789 (2019).

29. Li, M. X. et al. Highly oriented graphite aerogel fabricated by confined liquid-phase expansion for anisotropically thermally conductive epoxy composites. ACS Appl. Mater. Interfaces 12, 27476-27484 (2020).

30. Pang, K. et al. Hydroplastic foaming of graphene aerogels and artificially intelligent tactile sensors. Sci. Adv. 6, eabd4045 (2020).

31. Qiu, L., Liu, J. Z., Chang, S. L. Y., Wu, Y. Z. \& Li, D. Biomimetic superelastic graphene-based cellular monoliths. Nat. Commun. 3, 1241 (2012).

32. Chen, C. J. et al. Scalable and sustainable approach toward highly compressible, anisotropic, lamellar carbon Sponge. Chem 4, 544-554 (2018).

33. Yao, B. W., Chen, J., Huang, L., Zhou, Q. Q. \& Shi, G. Q. Base-induced liquid crystals of graphene oxide for preparing elastic graphene foams with long-range ordered microstructures. Adv. Mater. 28, 16231629 (2016).

34. Huang, M. et al. CVD growth of porous graphene foam in film form. Matter 3, 487-497 (2020).

35. Yang, H. S. et al. Superplastic air-dryable graphene hydrogels for wet-press assembly of ultrastrong superelastic aerogels with infinite macroscale. Adv. Funct. Mater. 29, 1901917 (2019).

36. Moon, I. K., Yoon, S., Chun, K. Y \& Oh, J. Highly elastic and conductive N-doped monolithic graphene aerogels for multifunctional applications. Adv. Funct. Mater. 25, 6976-6984 (2015).

37. Zhang, K. J. et al. Thermal and electrical transport in ultralow density single-walled carbon nanotube networks. Adv. Mater. 25, 2926-2931 (2013). 
38. Pettes, M. T., Ji, H. X., Ruoff, R. S. \& Shi, L. Thermal transport in three-dimensional foam architectures of few-layer graphene and ultrathin graphite. Nano Lett. 12, 2959-2964 (2012).

39. Menzel, R. et al. Joule heating characteristics of emulsion-templated graphene aerogels. Adv. Funct. Mater. 25, 28-35 (2015).

40. Xie, Y. S. et al. Graphene aerogel based bolometer for ultrasensitive sensing from ultraviolet to farinfrared, ACS Nano 13, 5385-5396 (2019).

41. Deng, X. et al. Nitrogen-doped unusually superwetting, thermally insulating and elastic graphene aerogel for efficient solar steam generation. ACS Appl. Mater. Interfaces 12, 26200-26212 (2020).

42. Poulaert, B. \& Issi, J. P. Low-temperature electrical resistivity of carbon-black-loaded polyethylene. Polymer 24, 841-845 (1983).

43. Xiu, H. et al. Formation of new electric double percolation via carbon black induced co-continuous like morphology. RSC Adv. 4, 37193-37196 (2014).

44 Huang, Y. et al. The influence of single-walled carbon nanotube structure on the electromagnetic interference shielding efficiency of its epoxy composites. Carbon 45, 1614-1621 (2007).

45. Kimura, T. et al. Polymer composites of carbon nanotubes aligned by a magnetic field. Adv. Mater. 14, 1380-1383 (2010).

46. Regev, O., ElKati, P. N. B., Loos, J. \& Koning, C. E. Preparation of conductive nanotube-polymer composites using latex technology. Adv. Mater. 16, 248-251 (2004).

47. Li, J. et al. Correlations between percolation threshold, dispersion state, and aspect ratio of carbon nanotubes. Adv. Funct. Mater. 17, 3207-3215 (2007).

48. Ram, R., Rahaman, M. \& Khastgir, D. Electrical properties of polyvinylidene fluoride (PVDF)/multiwalled carbon nanotube (MWCNT) semi-transparent composites: Modelling of DC conductivity. Composites Part A 69, 30-39 (2015).

49. Yousefi, N. et al. Highly aligned graphene/polymer nanocomposites with excellent dielectric properties for high-performance electromagnetic interference shielding. Adv. Mater. 26, 5480-5487 (2014).

50. Wu, C. et al. Highly conductive nanocomposites with three-dimensional, compactly interconnected graphene networks via a self-assembly process. Adv. Funct. Mater. 23, 506-513 (2013).

51. Long, G. C. et al. Resolving the dilemma of gaining conductivity but losing environmental friendliness in producing polystyrene/graphene composites via optimizing the matrix-filler structure. Green Chem. 15, 821-828 (2013). 
52. Chen, M. T. et al. Highly stretchable conductors integrated with a conductive carbon nanotube/graphene network and 3D porous poly(dimethylsiloxane). Adv. Funct. Mater. 24, 7548-7556 (2014).

53. Chen, Z. P., Xu, C., Ma, C. Q., Ren, W. C. \& Cheng, H. M. Lightweight and flexible graphene foam composites for high-performance electromagnetic interference shielding. Adv. Mater. 25, 1296-1300 (2013).

54. Liu, J. et al. Hydrophobic, flexible, and lightweight MXene foams for high-performance electromagnetic-interference shielding. Adv. Mater. 29, 1702367 (2017).

55. Han, M. K. et al. Anisotropic MXene aerogels with a mechanically tunable ratio of electromagnetic wave reflection to absorption. Adv. Optical Mater. 7, 1900267 (2019).

56. Zeng, Z. H. et al. Nanocellulose-MXene biomimetic aerogels with orientation-tunable electromagnetic interference shielding performance. Adv. Sci. 7, 2000979 (2020).

57. Sun, R. H. et al. Highly conductive transition metal carbide/carbonitride(MXene) @polystyrene nanocomposites fabricated by electrostatic assembly for highly efficient electromagnetic interference shielding. Adv. Funct. Mater. 27, 1702807 (2017).

58. Chen, Y. et al. High-performance epoxy nanocomposites reinforced with three-dimensional carbon nanotube sponge for electromagnetic interference shielding. Adv. Funct. Mater. 26, 447-455 (2016).

59. Zeng, Z. H. et al. Lightweight and anisotropic porous MWCNT/WPU composites for ultrahigh performance electromagnetic interference shielding. Adv. Funct. Mater. 26, 303-310 (2016).

60. Lu, D. W. et al. Flexible, lightweight carbon nanotube sponges and composites for high-performance electromagnetic interference shielding. Carbon 133, 457-463 (2018).

61. Zeng, Z. H. et al. Thin and flexible multi-walled carbon nanotube/waterborne polyurethane composites with high-performance electromagnetic interference shielding. Carbon 96, 768-777 (2016).

62. Zeng, Z. H. et al. Ultralight, flexible, and biomimetic nanocellulose/silver nanowire aerogels for electromagnetic interference shielding. ACS Nano 14, 2927-2938 (2020).

63. Zeng, Z. H. et al. Ultralight and flexible polyurethane/silver nanowire nanocomposites with unidirectional pores for highly effective electromagnetic shielding. ACS Appl. Mater. Interfaces 9 , 32211-32219 (2017).

64. Wan, Y. J. et al. Anticorrosive, ultralight, and flexible carbon-wrapped metallic nanowire hybrid sponges for highly efficient electromagnetic interference shielding. Small 14, 1800534 (2018). 


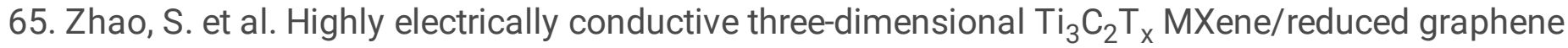
oxide hybrid aerogels with excellent electromagnetic interference shielding performances. ACS Nano 12, 11193-11202 (2018).

66. Song, Q. et al. Carbon nanotube-multilayered graphene edge plane core-shell hybrid foams for ultrahigh-performance electromagnetic-interference shielding. Adv. Mater. 29, 1701583 (2017).

67. Wei, Q. W. et al. Superhigh electromagnetic interference shielding of ultrathin aligned pristine graphene nanosheets film. Adv. Mater. 32, 1907411 (2020).

68. Yan, D. X. et al. Structured reduced graphene oxide/polymer composites for ultra-Efficient electromagnetic interference shielding. Adv. Funct. Mater. 25, 559-566 (2015).

\section{Figures}
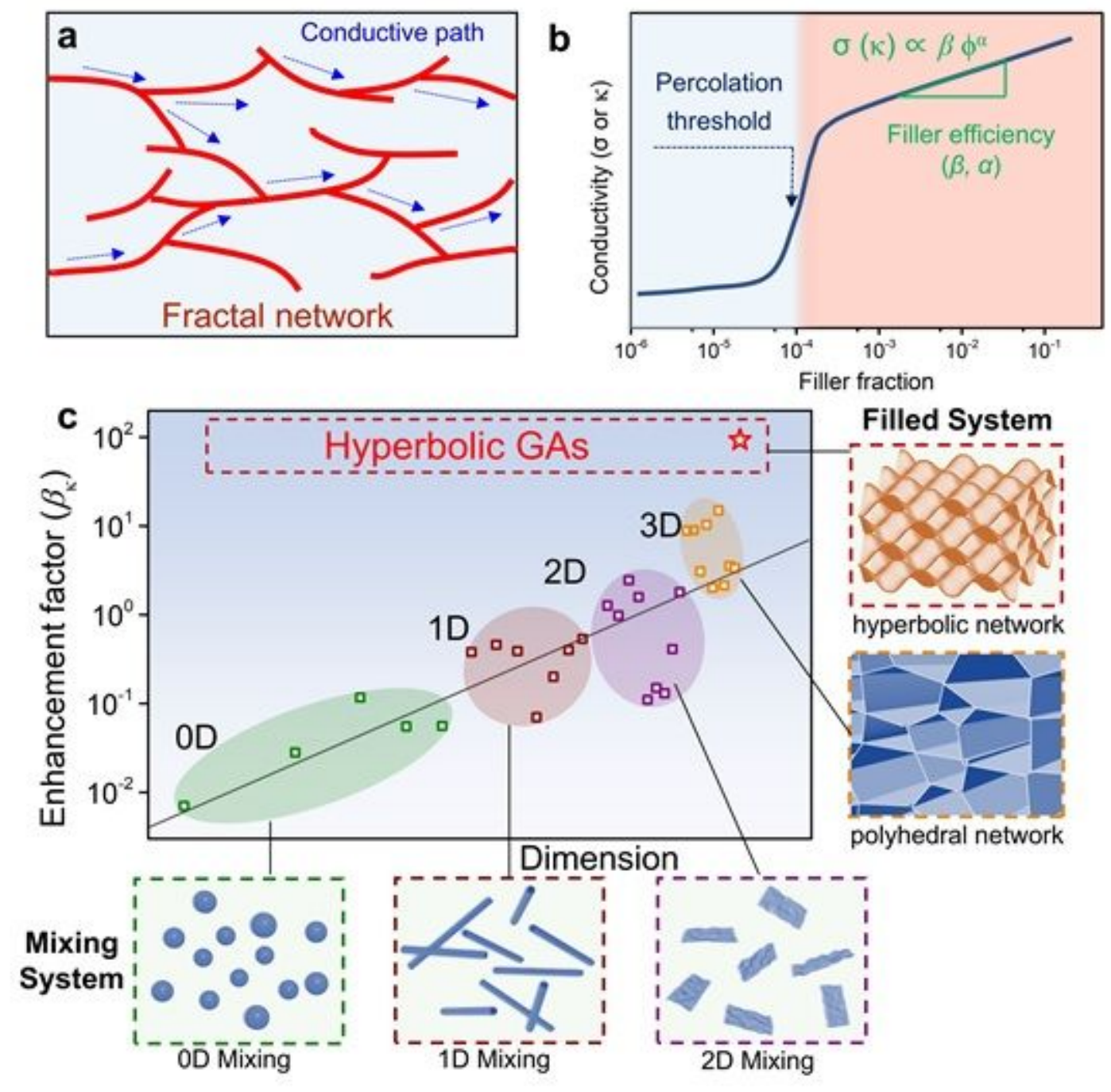

Figure 1 
Schematic illustrations of polymer nanocomposites with OD, 1D, 2D and 3D nanofillers. (a) Representative diagram of continuous conductive paths in polymer nanocomposite and (b) its typical evolution of conductive performances. (c) Thermal enhancement factor of polymer nanocomposites with different nanofillers, divided into mixing and filled systems.
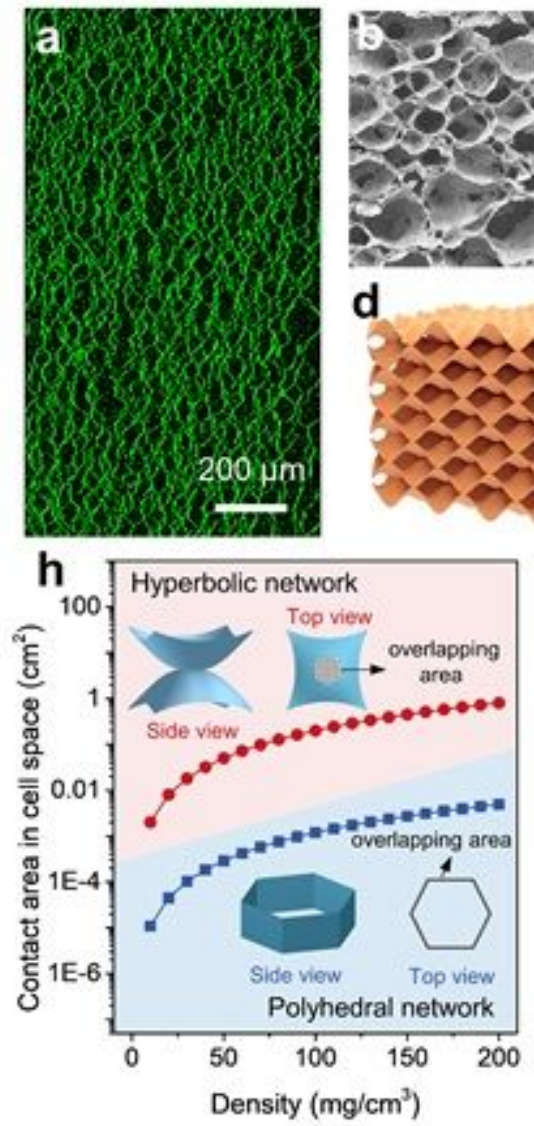

d
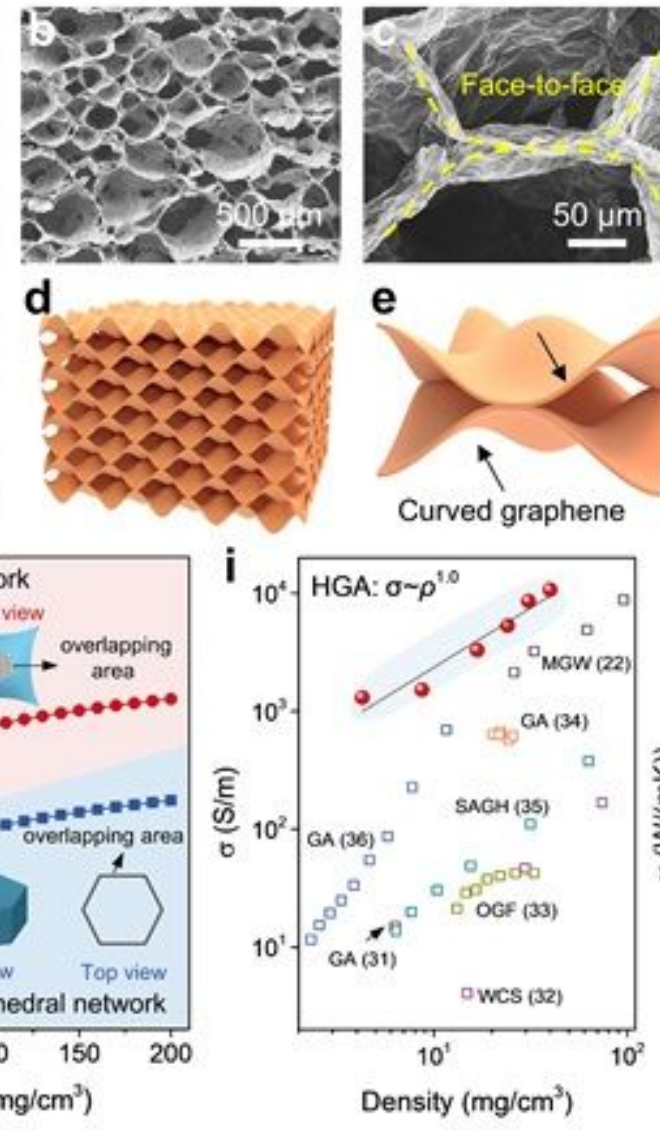
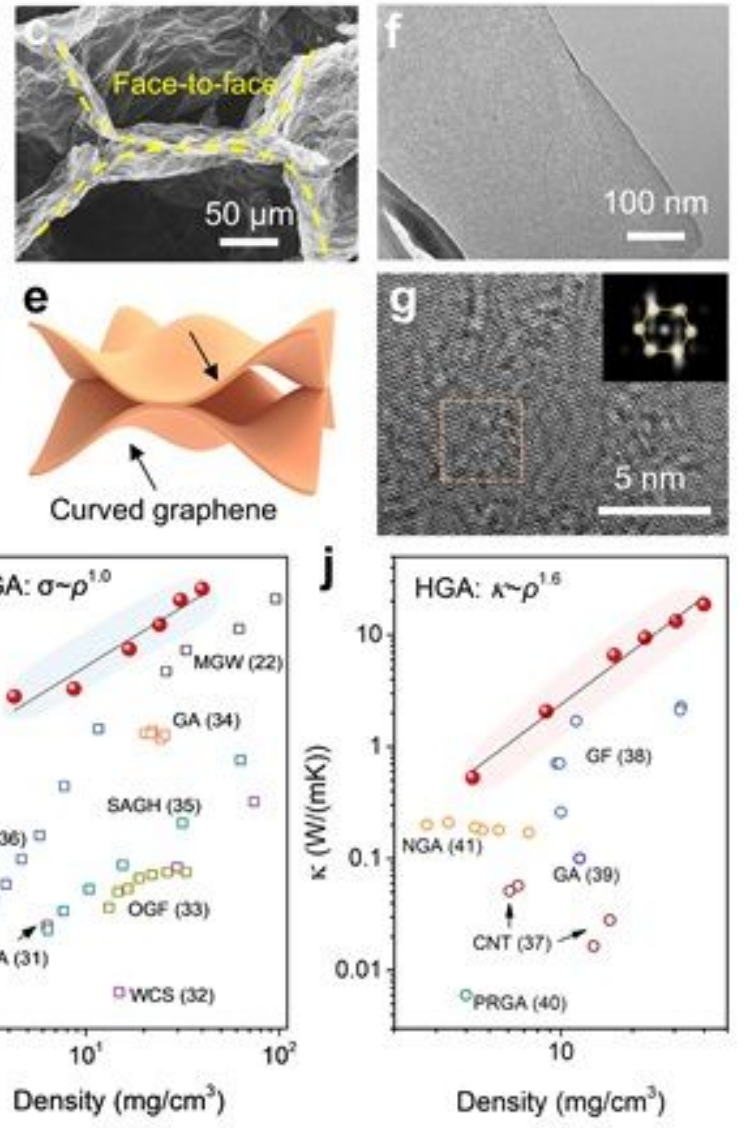

Figure 2

Morphology and physical properties of HGAs. (a) X-ray computed microtomography, (b and c) SEM images and ( $d$ and e) schematic diagrams of cross-sectional morphology of HGAs. (f) TEM and (g) HRTEM images of $\mathrm{HGAs}$ after $2,800^{\circ} \mathrm{C}$ heat treatment. The inset is electron diffraction pattern of the square region. (h) Theoretical calculation of contact area of hyperboloidal networks and polyhedral networks. The insets are side views and top views of the hyperbolic networks and polyhedral networks. The shaded area is the overlap area between the graphene sheets. ( $\mathrm{i}$ and $\mathrm{j}$ ) Comparisons of electrical conductivity and thermal conductivity of ever-reported carbon networks. 
a

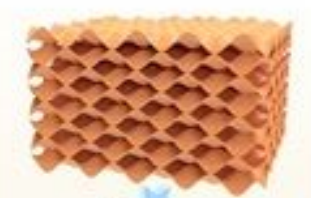

\section{亳重}

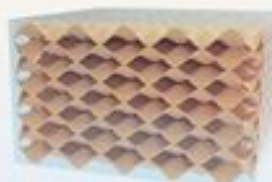

byy
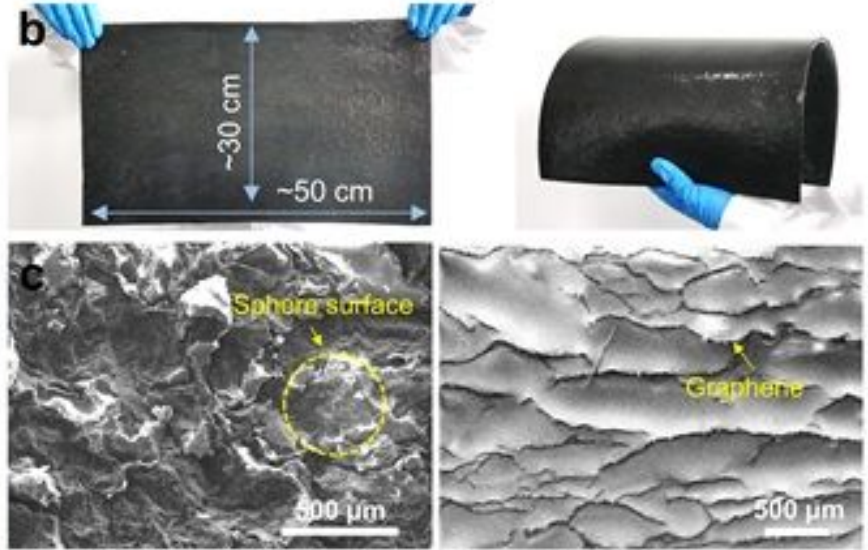

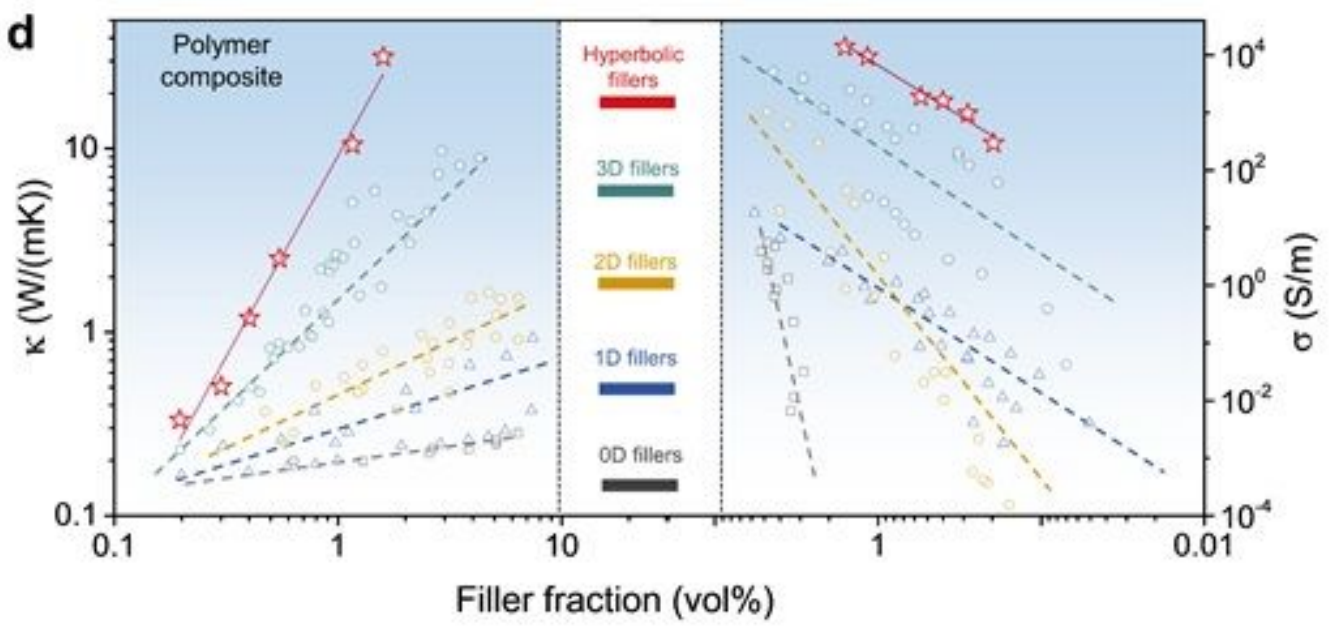

Figure 3

HGA filled polymer nanocomposites. (a) A schematic representation of the HGA embedment into PDMS matrix. (b) Digital photos of large-sized flexible PGC. (c) Top- and side-view SEM images of PGC with typical HGA tracks. (d) Comparisons of thermal conductivity and electrical conductivity of PGC with other reported polymer composites. 

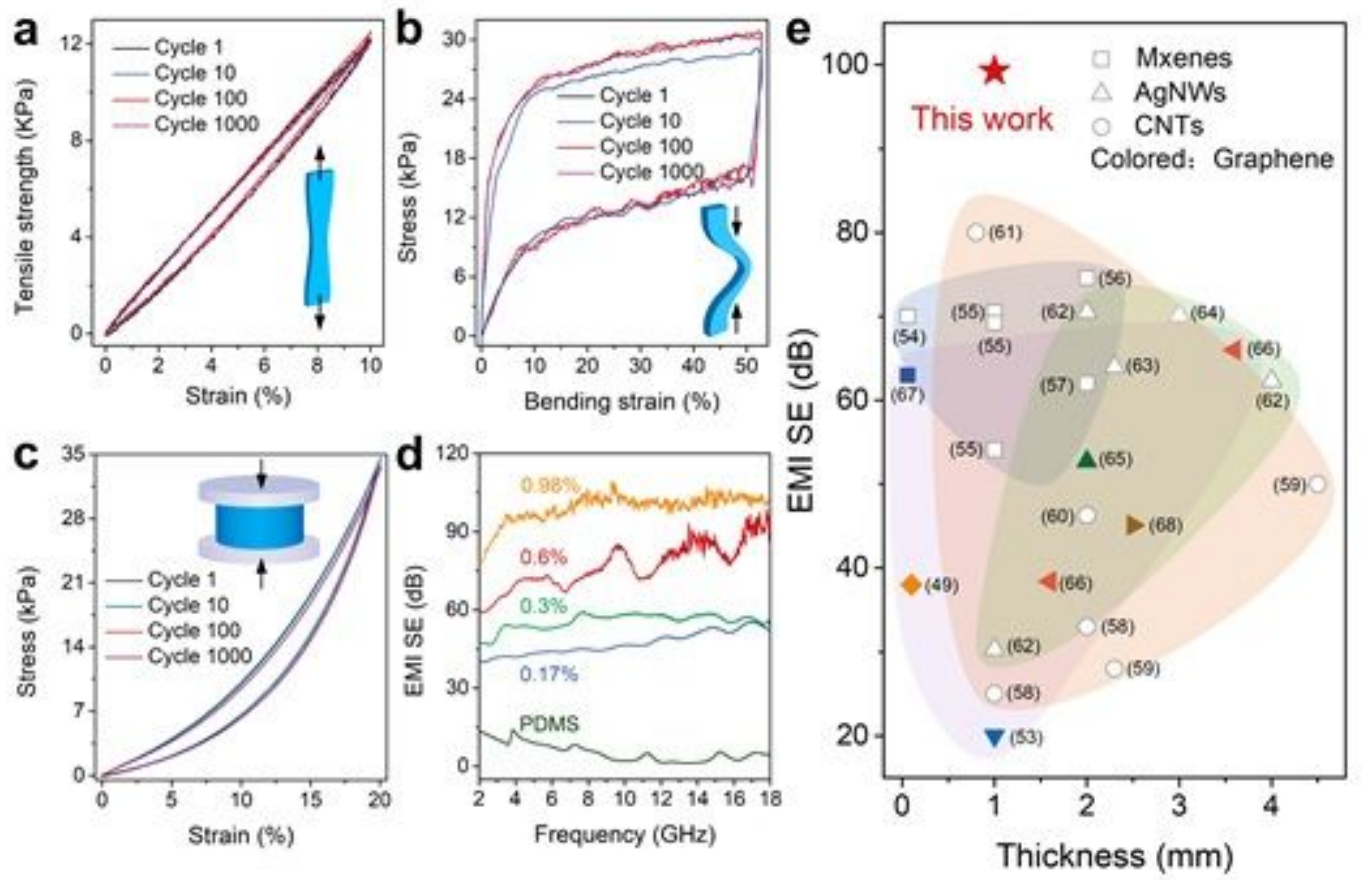

\section{Figure 4}

Mechanical and electromagnetic properties of PGCs. (a) Mechanical stretching, (b) bending and (c) compressive performances of PGCs for 1,000-cycle tests. (d) EMI shielding performance of PGCs with frequency range of 2-18 GHz at $1 \mathrm{~mm}$ thickness. (e) Comparison of EMI shielding performance between the PGCs and other polymer composites with nanofillers of MXenes, CNTs, AgNWs and graphene.
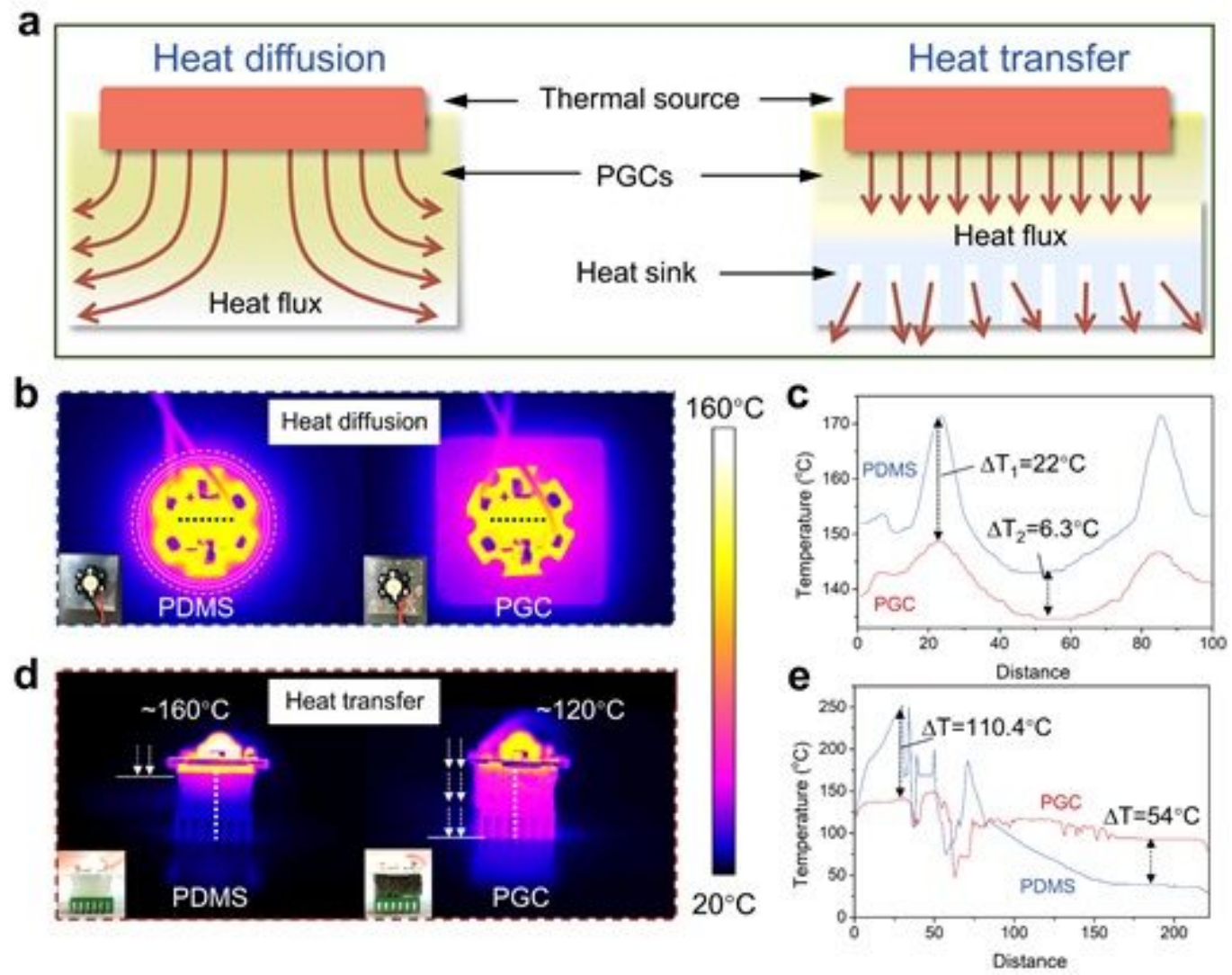


\section{Figure 5}

Thermal management of flexible PGC. (a) A schematic diagram of PGC as heat diffusion and heat transfer materials for a high-power LED lamp. (b) Infrared images of an LED lamp with PDMS and PGC for heat dissipation and (c) plotted temperature profiles. (d) Infrared images of a LED lamp with PDMS and PGC for heat transfer and (e) plotted temperature profiles.

\section{Supplementary Files}

This is a list of supplementary files associated with this preprint. Click to download.

- MovieS1.mov

- Movies2.mov

- SupplementaryMaterials.pdf 